\title{
尾叶樱桃天然种群叶表型性状变异研究
}

\author{
朱 弘朱淑霞李涌福伊贤贵段一凡王贤荣”
}

南京林业大学南方现代林业协同创新中心, 生物与环境学院, 亚热带森林生物多样性保护国家林草局重点实验室, 南京 210037

摘 要 为揭示中国特有植物尾叶樱桃(Cerasus dielsiana)在现代核心分布区天然种群的叶表型地理变异规律及其生态适应 性特征, 该研究通过多重比较、巢氏方差分析、相关性分析、主成分分析(PCA)、主坐标分析(PCoA)、非加权配对算术平均 法(UPGMA)聚类分析等数理方法, 对来自四川、湖北、湖南、江西、台湾 5 省 8 个尾叶樱桃天然种群的 11 个叶表型性状进行了 比较分析, 研究其不同地理单元间叶表型多样性和地理变异规律及对地理气候的响应。结果显示: 1)尾叶樱桃主要叶表型性 状变异在种群内和种群间均存在显著差异, 平均变异系数为 $22.44 \%$, 其中变异系数最大和最小的分别为叶面积 $(50.83 \%)$ 与一 级侧脉数 $(7.96 \%)$; 平均叶表型性状的分化系数为 $30.78 \%$, 种群内的变异 $(51.55 \%)$ 大于种群间的变异 $(22.55 \%)$ 。2) PCA表明对 尾叶樱桃叶表型性状变异起主要贡献作用的前三大主成分累计贡献率达到 $92.400 \%$, 可以综合概括和排序为“大小性状” (73.242\%)与 “形状性状” $(19.158 \%)$ 。3)叶宽 $(r=-0.641)$ 、叶面积 $(r=-0.658)$ 和一级侧脉数 $(r=0.659)$ 性状均与经度呈显著负相 关或正相关关系, 气温季节变化和最湿季降水量对叶表型性状变异影响较大。4)基于PCoA和UPGMA聚类分析可将 8 个天然 种群划分为4类。尾叶樱桃天然种群叶表型性状变异丰富, 在数量上表现出一定的连续性, “大小性状” 是性状间变异的主要来 源, 平均表型分化处于中等程度水平, 种群内是叶表型性状变异的主要来源; 各种群间表型分化划分结果与地理位置基本一 致, 在地理空间上呈现以经度为主的梯度变异模式, “气候变异性”与“展叶期降水量”是驱动叶表型性状变异的主要气候因子, 推测这是尾叶樱桃在长期进化中与亚热带季风气候环境相适应的结果。

关键词 尾叶樱桃; 天然种群; 地理-气候因子; 叶表型分化

朱弘, 朱淑霞, 李涌福, 伊贤贵, 段一凡, 王贤荣 (2018). 尾叶樱桃天然种群叶表型性状变异研究. 植物生态学报, 42, 1168-1178. DOI: 10.17521/cjpe.2018.0196

\section{Leaf phenotypic variation in natural populations of Cerasus dielsiana}

\section{ZHU Hong, ZHU Shu-Xia, LI Yong-Fu, YI Xian-Gui, DUAN Yi-Fan, and WANG Xian-Rong*}

Co-Innovation Center for Sustainable Forestry in Southern China, College of Biology and the Environment, Key Laboratory of State Forestry and Grassland Administration on Subtropical Forest Biodiversity Conservation, Nanjing Forestry University, Nanjing 210037, China

\begin{abstract}
Aims Cerasus dielsiana is a wild cherry species endemic to the subtropical forest of China, and was regarded as a promising ornamental resource. Our objectives were to determine the leaf phenotypic variation, adaptation and patterns in eight natural $C$. dielsiana populations.

Methods We analyzed eleven leaf phenotypic traits from five provinces of China in eight natural populations of C. dielsiana by using multiple comparisons, nested analysis of variance, correlation analysis, principal component analysis (PCA), principal coordinate analysis (PCoA) and unweighted pair-group method with arithmetic mean (UPGMA) cluster analysis.

Important findings Results showed that 1) Rich leaf phenotypic variation existed among and within populations, and the average coefficient of variation $(\mathrm{CV})$ was $22.44 \%$, the maximum and the minimum were leaf area $(C V=50.83 \%)$ and primary lateral veins $(C V=7.96 \%)$, respectively. The mean differentiation coefficient $\left(V_{\text {st }}\right)$ for all traits was $30.78 \%$, and the variation within populations $(51.55 \%)$ was higher than that among populations (22.55\%). 2) The principal component analysis showed that the cumulative contribution rate of the first three main components of variation from leaf phenotypic traits of $C$. dielsiana made a major contribution reached to $92.400 \%$, and can be comprehensively summarized and sorted as "size traits" $(73.242 \%)$ and "shape traits" (19.158\%). 3) Leaf width $(r=-0.641)$, leaf area $(r=-0.658)$ and primary lateral veins $(r=0.659)$ showed significant negative or positive correlation with longitude, and the temperature seasonality and precipitation of
\end{abstract}

收稿日期Received: 2018-08-10 接受日期Accepted: 2018-11-02

* 通信作者Corresponding author (wangxianrong66@njfu. edu.cn) 
wettest quarter were showed more influence on leaf phenotype variation. 4) The eight natural populations of $C$. dielsiana could be divided into four groups according to principal coordinate analysis (PCA) and UPGMA cluster analysis. To sum up, leaf phenotypic variation in C. dielsiana is abundant, with a certain of continuity in quantity, and "size trait" is the main source of inter-trait variation. The mean differentiation coefficient at a moderate level, the phenotypic variation within populations was the main source of leaf traits variation. The results of phenotypic differentiation among populations were found to be consistent with the geographical location, and presented a gradient variation pattern dominated by longitude geographically. Meanwhile, the "climate variability" and "leaf-expansion period" are the main climatic factors that drive leaf phenotypic variation. We speculate the phenomena results from a long evolutionary adaptation of $C$. dielsiana to the subtropical monsoon climate.

Key words Cerasus dielsiana; natural population; geography and climatic factors; leaf phenotypic differentiation

Zhu H, Zhu SX, Li YF, Yi XG, Duan YF, Wang XR (2018). Leaf phenotypic variation in natural populations of Cerasus dielsiana. Chinese Journal of Plant Ecology, 42, 1168-1178. DOI: 10.17521/cjpe.2018.0196

植物物种多样性研究有多种标记方式, 形态学 标记(表型)是其中的重要组成部分(方炎明和丁雨龙, 2016), 它主要研究同一物种不同种群在分布区内 不同环境条件下的变异样式, 相较于细胞学、生化 和分子标记手段, 具有测量简便、快速、经济的优 点。数量分类学是开展植物表型研究最主要的手段, 即通过有效的样品采样、合理的数理统计方法, 揭 示植物表型性状的变异大小和遗传规律, 客观地评 价遗传多样性, 已经被许多学者广泛应用于经济作 物(Ali, 2018)、林木(江锡兵等, 2013; Li et al., 2018)、 园艺品种(张往祥等, 2017)和入侵种(Wang et al., 2018)等研究中。其中植物叶表型性状不仅是植物分 类学中最直观与可操作的分类性状, 同时也反映了 植物对生长环境的响应和适应, 有助于认识环境篎 选过滤对植物功能性状的作用以及由此产生的物种 生物地理分布格局(Wright et al., 2007)。

尾叶樱桃(Cerasus dielsiana) 隶属蓄薇科樱属, 为我国特有落叶小乔木, 主要分布在我国亚热带季 风区的各省山区，分布海拔500-1400 m; 因其树形 优美、早春先花后叶、花色多样, 具有极高的观赏 价值, 加上生态适应性广、抗逆性强, 市场开发前景 十分广阔(王贤荣, 2014)。由于尾叶樱桃分布区生境 多样, 受局部地形及小气候的影响, 加上种间、种内 存在潜在的自然杂交, 导致该物种不同地理种群间 在区域尺度上产生了一定的形态分化, 目前尚无针 对尾叶樱桃天然种群地理变异、叶表型多样性的系 统报道。本文通过对区域尺度的尾叶樱桃天然分布 区8 个代表种群的叶表型性状多样性进行初步评估, 从形态学水平上揭示该种种群内和种群间地理变异 样式, 探明叶表型性状与地理-气候因子的相关关 系, 研究结果可为今后开展尾叶樱桃分类鉴定、种
质资源收集保育与开发利用提供理论依据。

\section{1 材料和方法}

\section{1 气象和地理资料收集}

本研究的气候数据来源于世界气候数据库 WorldClim (http://www.worldclim.org/), 主要是根据 1950-2000年世界各地气象站的气候观测记录, 采 用插值法生成的空间分辨率为 $2.5^{\prime}$ (约 $5 \mathrm{~km}^{2}$ ) 的全球 气候数据包; 各种群的经度、纬度、海拔等地理信 息由手持GPS仪(UNI-T UT379B, 优利德, 广东东 莞)野外测定。利用DIVA-GIS软件(Hijmans et al., 2001) 提取 8 个研究区对应的 19 个通用气候变量 (bio1-bio19)。为减少各变量间共线性带来的干扰, 参照朱弘等(2017)的方法, 对所有环境变量做主成 分分析(PCA)降维处理, 最终篮选出代表第1主成分 (贡献率达 $97.459 \%$ ) 的4个变量，即bio4、bio12、bio16 和bio18作为影响尾叶樱桃种群分布的主导气候因 子用于后续分析(表1)。

\section{2 材料来源与样品采集}

表型性状测量材料为课题组在2014-2018年春 季全面开展野外调查、种群采样基础上获得的凭证 标本; 为了尽可能反映各分布区及其生态环境的 特点, 自西向东选出代表核心分区的四川(峨眉山) 1 个种群, 湖北(神农架、星斗山)、湖南(张家界、 莽山)、江西(金竹飞瀑、仙姑坛)各2个种群和新记录 的台湾(阿里山) 1 个种群, 共计 8 个种群; 由于种群数 量较小及取样困难, 每个种群随机选取 5 株发育良好 的代表株, 单株间的水平距离尽量在 $30 \mathrm{~m}$ 以上, 以减 少亲缘关系的影响; 选择每个单株阳面中部以上完 整无损的 1 个枝条作为凭证标本, 每份标本中再随机 各取 5 枚完整无损的成龄叶片, 每个种群共计 25 枚。 
取样种群的地理位置及主导气候因子特征详见表 2 。

\section{3 叶表型性状的选取与测量}

将尾叶樱桃 8 个种群的 200 枚叶片利用佳能Lide 120 扫描仪(Canon, Bắc Ninh, Vietnam)逐一进行扫描, 参考戴志聪等(2009)的方法对获得的电子图像利用 ImageJ 1.52c 软件(https://imagej.nih.gov/ij/download. $h t m l$ 测定。参考王贤荣等(2007)对樱属表型研究的 方法, 共选取 11 个参数, 其中直接测定的指标有 8 个, 分别为叶片长(叶基至叶尖距离)、叶片宽(叶片最宽 处距离)、叶柄长、叶面积、叶周长、一级侧脉数(到 达锯齿顶端的左右两侧叶脉总数)、叶尖夹角和叶基 夹角。再根据上述指标, 分别计算叶长宽比 $=$ 叶片长 $/$ 叶片宽、叶形指数 $=4 \pi \times$ 叶面积/叶周长平方和、叶 脉密度 $=$ 一级侧脉数 $/ 2$ 倍叶片长 3 个指标 $($ 李梅等, 2005; Meng et al., 2017)。

\section{4 数据统计分析方法}

观测记载的数据在Excel 2007软件中进行整理 汇总, 根据各性状 25 个样品的平均值 $(\bar{X})$ 和标准偏 差 $(S D)$ 计算变异系数 $(C V=$ 标准偏差/平均值); 采用 SPSS 16.0软件中的单因素方差分析(显著性检验采 用多重比较Duncan法), 研究叶表型性状在不同种
群间的变异水平; 采用巢式方差设计(李斌等, 2002) 进一步探讨比较尾叶樱桃叶表型11个性状在种群间 和种群内的来源和分布状况, 其线性模型为: $Y_{i j k}=$ $\mu+S_{i}+T_{(i) j}+e_{(i j) k}$ 。其中 $Y_{i j k}$ 为第 $i$ 个种群第 $j$ 个单株第 $k$ 个观测值; $\mu$ 为总体均值; $S_{i}$ 为种群间的效应(固定); $T_{(i) j}$ 为种群内单株效应 $\left(\right.$ 随机); $e_{(i j) k}$ 为随机误差。为了 与遗传分化系数 $\left(G_{\mathrm{st}}\right)$ 相对应，参照葛颂等(1988)的 表型分化系数公式: $V_{\mathrm{st}}=\delta_{\mathrm{t} / \mathrm{s}}^{2} /\left(\delta_{\mathrm{t} / \mathrm{s}}^{2}+\delta_{\mathrm{s}}^{2}\right)$, 其中 $\left(V_{\mathrm{st}}\right)$ 为 表型分化系数, 表示种群间变异占遗传总变异的比 例，其中 $\delta_{\mathrm{t} / \mathrm{s}}^{2}$ 为种群间方差分量， $\delta_{\mathrm{s}}^{2}$ 为种群内方差分 量。再通过Person相关分析及单尾检验得出叶表型 性状对地理气候因子的响应, 为保证数据符合正态 分布和方差齐性，先对原始数据进行标准化处理; 通过PCA, 得出不同表型性状对种群变异贡献率; 上述分析在SPSS 16.0软件中完成; 为了更直观地反 映各种群遗传距离远近, 采用PAST3 3.2 软件包 (http://folk.uio.no/ohammer/past/)绘制数据标准化后 的基于环境变量与各性状平均值的联合矩阵的主坐 标分析(PCoA) 图和采用非加权配对算术平均法 (UPGMA)的聚类分析图, 其中靴带值验证(bootstrap test) 1000 次。

表1 主成分分析(PCA)斾选后影响尾叶樱桃的 4 个主导气候变量及其贡献率

Table 1 Four dominant climatic variables that affect Cerasus dielsiana after principal component analysis (PCA) screening and its contributions

\begin{tabular}{llcc}
\hline $\begin{array}{l}\text { 代码 } \\
\text { Code }\end{array}$ & $\begin{array}{l}\text { 气候变量 } \\
\text { Climatic variables }\end{array}$ & $\begin{array}{c}\text { 特征值 } \\
\text { Eigenvalue }\end{array}$ & $\begin{array}{c}\text { 作为第1主成分的贡献率 } \\
\text { Variance contribution rate as first principal component (\%) }\end{array}$ \\
\hline bio4 & 气温季节变化 Temperature seasonality $(S D \times 100)$ & -0.151 & $97.459 \%$ \\
bio12 & 年降水量 Annual precipitation $(\mathrm{mm})$ & 0.784 & 0.420 \\
bio16 & 最湿季降水量 Precipitation of wettest quarter $(\mathrm{mm})$ & 0.403 & \\
bio18 & 最暖季降水量 Precipitation of warmest quarter $(\mathrm{mm})$ & & \\
\hline
\end{tabular}

表2 尾叶樱桃8个天然种群的地理位置及主导气候因子特征

Table 2 Geographical locations of the eight natural populations of Cerasus dielsiana and characters of dominant climatic factors

\begin{tabular}{|c|c|c|c|c|c|c|c|}
\hline $\begin{array}{l}\text { 种群 } \\
\text { Population }\end{array}$ & $\begin{array}{c}\text { 纬度 } \\
\text { Latitude } \\
(\mathrm{N})\end{array}$ & $\begin{array}{c}\text { 经度 } \\
\text { Longitude } \\
\text { (E) }\end{array}$ & $\begin{array}{c}\text { 平均海拔 } \\
\text { Mean } \\
\text { altitude (m) }\end{array}$ & $\begin{array}{c}\text { 气温季节变化 } \\
\text { Temperature } \\
\text { Seasonality } \\
\text { bio4 }(S D \times 100)\end{array}$ & $\begin{array}{c}\text { 年降水量 } \\
\text { Annual } \\
\text { precipitation } \\
\text { bio12 }(\mathrm{mm})\end{array}$ & $\begin{array}{c}\text { 最湿季降水量 } \\
\text { Precipitation of } \\
\text { wettest quarter } \\
\text { bio16 (mm) }\end{array}$ & $\begin{array}{c}\text { 最暖季降水量 } \\
\text { Precipitation of } \\
\text { warmest quarter } \\
\text { bio18 (mm) }\end{array}$ \\
\hline 四川峨眉山 Mt. Emei, Sichuan (EMS) & $29.57^{\circ}$ & $103.37^{\circ}$ & 1365 & 7.53 & 1264.00 & 92.13 & 710.00 \\
\hline 湖北神农架 Shennongjia, Hubei (SNJ) & $31.45^{\circ}$ & $110.25^{\circ}$ & 860 & 8.15 & 1696.00 & 51.61 & 557.00 \\
\hline 湖北星斗山 Mt. Xingdou, Hubei (XDS) & $30.02^{\circ}$ & $109.12^{\circ}$ & 839 & 8.19 & 1772.00 & 60.22 & 568.00 \\
\hline 湖南莽山 Mt. Mangshan, Hunan (MS) & $24.97^{\circ}$ & $112.88^{\circ}$ & 1228 & 7.66 & 1492.00 & 56.44 & 601.00 \\
\hline 湖南张家界 Zhangjiajie, Hunan (ZJJ) & $29.32^{\circ}$ & $110.42^{\circ}$ & 1118 & 7.91 & 1591.00 & 62.14 & 556.00 \\
\hline 江西金竹飞瀑 Jinzhufeipu, Jiangxi (JZFP) & $27.00^{\circ}$ & $115.92^{\circ}$ & 966 & 7.20 & 1508.00 & 60.26 & 609.00 \\
\hline 江西仙姑坛 Xiangutan, Jiangxi (XGT) & $28.45^{\circ}$ & $114.38^{\circ}$ & 1364 & 6.86 & 1405.00 & 62.72 & 591.00 \\
\hline 台湾阿里山 Mt. Ali, Taiwan (ALS) & $23.50^{\circ}$ & $120.80^{\circ}$ & 1182 & 8.20 & 3747.00 & 80.66 & 1780.00 \\
\hline
\end{tabular}




\section{2 结果和分析}

\section{1 尾叶樱桃叶表型性状变异特征}

从尾叶樱桃叶表型性状在各种群间的Duncan 多重比较可以看出, 叶片最宽、叶柄最长、叶面积 最大、叶周长最长、密度最小的均来自四川峨眉山 种群; 叶形指数最大、一级侧脉数最多、叶尖夹角 最大、叶基夹角最大、叶长宽比最小的均来自台湾 阿里山种群; 叶长次长、叶长宽比最长、叶基夹角 次小的来自湖南莽山种群; 叶形指数最小、叶尖夹 角最小、一级侧脉数最多的均来自江西仙姑坛种群; 叶宽最短、叶周长最短、叶尖夹角最小、叶脉密度 最大的均来自江西金竹飞瀑种群; 各叶表型性状在 种群间显著差异, 但总体表现为连续变异。11个叶 表型性状平均 $C V$ 为 $22.44 \%, C V$ 最大的为叶面积 $(C V$ $=50.83 \%)$, 最小的为一级侧脉数 $(C V=7.96 \%)$; 通 过变异系数的比较, 可以发现 11 个叶表型性状在种 群间的差异程度依次为: 叶面积 $>$ 叶柄长 $>$ 叶宽 $>$ 叶 脉密度 $>$ 叶周长 $>$ 叶长 $>$ 叶尖夹角 $>$ 叶长宽比 $>$ 叶基夹 角 $>$ 叶形指数 $>$ 一级侧脉数 (表 3 )。

利用巢氏方差对 11 个尾叶樱桃叶表型性状在种 群间和种群内的差异检验结果表明，除一级侧脉数
和叶基夹角外, 其余在两个层次上均达到极显著差 异 $(p<0.01)$, 表明变异广泛(表4)。

\section{2 尾叶樱桃叶表型变异来源及种群间性状分化}

巢氏方差分析结果表明, 种群间表型分化系数 的变化范围是 9.61\%-66.69\%, 平均叶表型性状的分 化系数为 $30.78 \%$, 其中叶基夹角 $(66.69 \%)$ 的表型分 化系数最大, 其次为一级侧脉数 $(58.23 \%)$ 与叶柄长 (48.47\%), 而叶尖夹角 $(9.61 \%)$ 的表型分化系数最小; 11 个叶表型性状在种群内和种群间的平均方差分量 百分比分别为 $51.55 \%$ 和 $22.55 \%$, 种群内的方差分量 远大于种群间的方差分量, 说明种群内的变异是尾 叶樱桃叶表型变异的主要来源(表5)。

\section{3 尾叶樱桃叶表型性状的主成分分析}

主成分分析结果表明, 11 个叶表型性状中的前3 个主成分方差贡献率分别为 $73.242 \% 、 11.319 \%$ 和 $7.839 \%$ ，累计贡献率达到 $92.400 \%$ ，能够代表叶表 型特征变量的绝大部分信息; 第 1 主成分主要表征 为“大小性状”，包含叶面积、叶宽、叶周长、叶长 和叶脉密度; 第2和第3主成分主要表征为“形状性 状”，分别包含叶柄长和叶尖夹角，叶长宽比、叶形 指数和叶基夹角(表 6$)$ 。

表3 尾叶樱桃 8 个天然种群 11 个叶表型性状的变异情况

Table 3 Variations of eleven leaf phenotypic traits among eight natural populations of Cerasus dielsiana

\begin{tabular}{|c|c|c|c|c|c|c|c|c|c|c|}
\hline \multirow{2}{*}{$\begin{array}{l}\text { 叶表型性状 } \\
\text { Leaf phenotypic } \\
\text { trait }\end{array}$} & \multicolumn{10}{|c|}{ 种群 Population } \\
\hline & EMS & SNJ & XDS & MS & ZJJ & JZFP & XGT & ALS & $\begin{array}{l}\text { 平均值 } \\
\text { Mean }\end{array}$ & $\begin{array}{c}\text { 变异系数 } \\
C V(\%)\end{array}$ \\
\hline 叶长 $L L(\mathrm{~mm})$ & $88.80 \pm 22.85^{\mathrm{a}}$ & $79.08 \pm 8.62^{\mathrm{b}}$ & $\begin{array}{l}83.40 \pm \\
17.18^{\mathrm{ab}}\end{array}$ & $87.48 \pm 11.27^{\mathrm{a}}$ & $89.63 \pm 12.56^{\mathrm{a}}$ & $54.57 \pm 12.41^{d}$ & $62.87 \pm 8.19^{\mathrm{c}}$ & $69.17 \pm 9.14^{\mathrm{c}}$ & 76.94 & 23.61 \\
\hline 叶宽 $L W(\mathrm{~mm})$ & $43.95 \pm 8.73^{\mathrm{a}}$ & $37.15 \pm 5.44^{\mathrm{bc}}$ & $33.90 \pm 9.02^{\mathrm{c}}$ & $34.93 \pm 4.96^{\mathrm{bc}}$ & $38.92 \pm 8.12^{\mathrm{b}}$ & $22.64 \pm 6.34^{\mathrm{e}}$ & $26.68 \pm 4.44^{\mathrm{d}}$ & $37.43 \pm 3.93^{\mathrm{bc}}$ & 34.45 & 26.39 \\
\hline 叶长宽比 $L W R$ & $2.01 \pm 0.21^{\mathrm{c}}$ & $2.15 \pm 0.19^{\mathrm{c}}$ & $2.50 \pm 0.27^{\mathrm{ab}}$ & $2.53 \pm 0.35^{\mathrm{a}}$ & $2.36 \pm 0.38^{\mathrm{b}}$ & $2.44 \pm 0.27^{\mathrm{ab}}$ & $2.37 \pm 0.19^{\mathrm{b}}$ & $1.86 \pm 0.14^{\mathrm{d}}$ & 2.28 & 14.93 \\
\hline $\begin{array}{l}\text { 叶柄长 } \\
P L(\mathrm{~mm})\end{array}$ & & $8.86 \pm 1.49^{\mathrm{b}}$ & $7.59 \pm 2.46^{\mathrm{cd}}$ & $11.10 \pm 2.11^{\mathrm{a}}$ & $8.25 \pm 1.60^{\mathrm{bc}}$ & & $8.53 \pm 0.89^{\mathrm{bc}}$ & & 8.80 & 29.92 \\
\hline $\begin{array}{l}\text { 叶面积 } \\
L A\left(\mathrm{~mm}^{2}\right)\end{array}$ & & $\begin{array}{c}1870.77 \pm \\
476.40^{\mathrm{bc}}\end{array}$ & $\begin{array}{l}2045.84 \pm \\
1040.24^{\mathrm{bc}}\end{array}$ & & $\begin{array}{c}2306.43 \pm \\
829.25^{\mathrm{a}}\end{array}$ & & & & 1846.13 & 50.83 \\
\hline $\begin{array}{l}\text { 叶周长 } \\
L P(\mathrm{~mm})\end{array}$ & $213.94 \pm 50.90^{\mathrm{a}}$ & $\begin{array}{c}183.08 \pm \\
21.64^{\text {cd }}\end{array}$ & $\begin{array}{c}191.98 \pm \\
44.07^{\mathrm{bc}}\end{array}$ & & $\begin{array}{c}207.05 \pm \\
32.44^{\mathrm{ab}}\end{array}$ & & & & 179.81 & 23.77 \\
\hline 叶形指数 $L I$ & $0.73 \pm 0.04^{b}$ & $0.69 \pm 0.05^{\mathrm{c}}$ & $0.65 \pm 0.05^{\mathrm{d}}$ & $0.63 \pm 0.06^{\mathrm{d}}$ & $0.65 \pm 0.07^{\mathrm{d}}$ & $0.64 \pm 0.06^{\mathrm{d}}$ & $0.63 \pm 0.03^{\mathrm{d}}$ & $0.77 \pm 0.03^{\mathrm{a}}$ & 0.67 & 8.90 \\
\hline $\begin{array}{l}\text { 一级侧脉数 } \\
P L V\end{array}$ & $11.00 \pm 0.46^{\mathrm{c}}$ & $12.00 \pm 0.71^{\mathrm{b}}$ & $12.00 \pm 1.32^{\mathrm{b}}$ & $12.00 \pm 0.44^{\mathrm{b}}$ & $12.00 \pm 0.70^{\mathrm{b}}$ & $12.00 \pm 0.77^{\mathrm{b}}$ & $13.00 \pm 0.46^{\mathrm{a}}$ & $13.00 \pm 0.34^{\mathrm{a}}$ & 11.88 & 7.96 \\
\hline 叶脉密度 $V D$ & $0.06 \pm 0.01^{\mathrm{d}}$ & $0.07 \pm 0.01^{\mathrm{c}}$ & $0.07 \pm 0.01^{\mathrm{c}}$ & $0.07 \pm 0.01^{\mathrm{cd}}$ & $0.07 \pm 0.03^{\text {cd }}$ & $0.11 \pm 0.01^{\mathrm{a}}$ & $0.10 \pm 0.01^{b}$ & $0.10 \pm 0.01^{\mathrm{b}}$ & 0.08 & 24.42 \\
\hline $\begin{array}{l}\text { 叶尖夹角 } \\
A A\left({ }^{\circ}\right)\end{array}$ & $27.57 \pm 3.42^{\mathrm{cd}}$ & $28.99 \pm 3.5^{\mathrm{bc}}$ & $28.54 \pm 4.36^{\mathrm{bc}}$ & $24.56 \pm 2.79^{\mathrm{e}}$ & $31.02 \pm 5.67^{\mathrm{b}}$ & $25.22 \pm 7.02^{\mathrm{de}}$ & $22.99 \pm 2.64^{\mathrm{e}}$ & $40.24 \pm 4.46^{\mathrm{a}}$ & 28.64 & 22.87 \\
\hline $\begin{array}{l}\text { 叶基夹角 } \\
B A\left(^{\circ}\right)\end{array}$ & $114.47 \pm 14.05^{\mathrm{b}}$ & $\begin{array}{c}114.88{ }^{ \pm} \\
11.04^{\mathrm{b}}\end{array}$ & $\begin{array}{l}113.87 \pm \\
14.78^{\mathrm{b}}\end{array}$ & $\begin{array}{c}128.34 \pm \\
8.24^{\mathrm{a}}\end{array}$ & $\begin{array}{c}103.60 \pm \\
9.34^{c}\end{array}$ & $\begin{array}{c}121.53 \pm \\
17.54^{\mathrm{b}}\end{array}$ & $\begin{array}{l}116.68 \pm \\
15.03^{\mathrm{b}}\end{array}$ & $\begin{array}{c}130.67 \pm \\
13.33^{\mathrm{a}}\end{array}$ & 116.72 & 13.31 \\
\hline 平均值 Mean & - & - & - & - & - & - & - & - & - & 22.44 \\
\hline
\end{tabular}

$\mathrm{ALS}$, 台湾阿里山; EMS, 四川峨眉山; JZFP, 江西金竹飞瀑; MS, 湖南莽山; SNJ, 湖北神农架; XDS, 湖北星斗山; XGT, 江西仙姑坛; ZJJ, 湖南张家 界。同一行不同小写字母表示差异显著 $(p<0.05$, Duncan法)。

ALS, Mt. Ali, Taiwan. EMS, Mt. Emei, Sichuan; JZFP, Jinzhufeipu, Jiangxi; MS, Mt. Mangshan, Hunan; SNJ, Shennongjia, Hubei; XDS, Mt. Xingdou; Hubei; XGT, Xiangutan, Jiangxi; ZJJ, Zhangjiajie, Hunan. Different lowercase letters in the same line indicate significant difference $(p<0.05$, Duncan's significant difference test). $A A$, apex angle; $B A$, base angle; $L A$, leaf area; $L I$, leaf index; $L L$, leaf length; $L P$, leaf perimeter; $L W$, leaf width; $L W R$, length to width ratio; $P L$, petiole length; $P L V$, primary lateral veins; $V D$, vein density. 
表4 尾叶樱桃8个天然种群 11 个叶表型性状的巢氏方差分析

Table 4 Nested variance analysis of eleven leaf phenotypic traits among eight natural populations of Cerasus dielsiana

\begin{tabular}{|c|c|c|c|c|c|}
\hline \multirow{3}{*}{$\begin{array}{l}\text { 叶表型性状 } \\
\text { Leaf phenotypic } \\
\text { trait }\end{array}$} & \multicolumn{3}{|c|}{ 均方 Mean square } & \multicolumn{2}{|c|}{$F$ 值 $F$ value } \\
\hline & 种群间 & 种群内 & 随机误差 & 种群间 & 种群内 \\
\hline & Among polulations & Within polulations & Random errors & Among polulations & Within polulations \\
\hline$L L(\mathrm{~mm})$ & 174.11 & 333.09 & 203.98 & $23.41^{* *}$ & $44.79^{* *}$ \\
\hline$L W(\mathrm{~mm})$ & 41.10 & 83.58 & 47.29 & $27.00^{* *}$ & $54.91^{* *}$ \\
\hline$L W R$ & 0.16 & 0.12 & 0.07 & $21.94^{* *}$ & $16.46^{* *}$ \\
\hline$P L(\mathrm{~mm})$ & 6.80 & 18.95 & 4.21 & $19.45^{* *}$ & $54.20^{* *}$ \\
\hline$L A\left(\mathrm{~mm}^{2}\right)$ & 442870.00 & 889674.00 & 636437.00 & $16.55^{* *}$ & $33.25^{* *}$ \\
\hline$L P(\mathrm{~mm})$ & 262.46 & 1858.26 & 1160.49 & $22.60^{* *}$ & $117.18^{* *}$ \\
\hline$L I$ & 0.01 & 0.00 & 0.00 & $24.74^{* *}$ & $11.63^{* *}$ \\
\hline$P L V$ & 3.46 & 0.84 & 0.47 & $23.06^{* *}$ & 5.60 \\
\hline$V D$ & 0.00 & 0.00 & 0.00 & $43.74^{* *}$ & $55.25^{* *}$ \\
\hline$A A\left({ }^{\circ}\right)$ & 30.31 & 42.98 & 19.13 & $39.61^{* *}$ & $56.17^{* *}$ \\
\hline$B A\left({ }^{\circ}\right)$ & 905.20 & 227.54 & 159.85 & $10.97^{* *}$ & 2.76 \\
\hline
\end{tabular}

$A A$, 叶尖夹角; $B A$, 叶基夹角。 $L A$, 叶面积; $L I$, 叶形指数; $L L$, 叶长; $L P$, 叶周长; $L W$, 叶宽; $L W R$, 叶长宽比; $P L$, 叶柄长; $P L V$, 一级侧脉数; $V D$, 叶脉 密度; ${ }^{* *}, p<0.01$ 。

$A A$, apex angle; $B A$, base angle. $L A$, leaf area; $L I$, leaf index; $L L$, leaf length; $L P$, leaf perimeter; $L W$, leaf width; $L W R$, length to width ratio; $P L$, petiole length; $P L V$, primary lateral veins; $V D$, vein density. ${ }^{* *}, p<0.01$.

表5 尾叶樱桃叶表型性状的方差分量与表型分化系数

Table 5 Variance component and phenotypic differentiation coefficient of leaf phenotypic traits among Cerasus dielsiana populations

\begin{tabular}{|c|c|c|c|c|c|c|c|}
\hline \multirow{2}{*}{$\begin{array}{l}\text { 叶表型性状 } \\
\text { Leaf phenotypic } \\
\text { trait }\end{array}$} & \multicolumn{3}{|c|}{ 方差分量 Variance component } & \multicolumn{3}{|c|}{ 方差分量百分比 Percentage of variance component (\%) } & \multirow{2}{*}{$\begin{array}{l}\text { 表型分化系数 } \\
\text { Phenotype } \\
\text { differentiation } \\
\text { coefficient (\%) }\end{array}$} \\
\hline & $\begin{array}{c}\text { 种群间 } \\
\text { Among populations }\end{array}$ & $\begin{array}{c}\text { 种群内 } \\
\text { Within populations }\end{array}$ & $\begin{array}{c}\text { 随机误差 } \\
\text { Random errors }\end{array}$ & $\begin{array}{c}\text { 种群间 } \\
\text { Among populations }\end{array}$ & $\begin{array}{c}\text { 种群内 } \\
\text { Within populations }\end{array}$ & $\begin{array}{c}\text { 随机误差 } \\
\text { Random Errors }\end{array}$ & \\
\hline$L L(\mathrm{~mm})$ & 6.359 & 25.822 & 13.324 & 13.98 & 56.75 & 29.28 & 19.76 \\
\hline$L W(\mathrm{~mm})$ & 1.699 & 7.259 & 3.343 & 13.81 & 59.01 & 27.18 & 18.97 \\
\hline$L W R$ & 0.002 & 0.001 & 0.005 & 29.27 & 60.67 & 29.27 & 14.22 \\
\hline$P L(\mathrm{~mm})$ & 0.486 & 0.517 & 0.272 & 38.13 & 40.54 & 21.33 & 48.47 \\
\hline$L A\left(\mathrm{~mm}^{2}\right)$ & 17872.160 & 50647.400 & 35586.960 & 17.17 & 48.65 & 34.18 & 26.08 \\
\hline$L P(\mathrm{~mm})$ & 63.832 & 139.554 & 74.330 & 22.98 & 50.25 & 26.76 & 31.38 \\
\hline$L I$ & 0.000 & 0.001 & 0.000 & 22.49 & 55.32 & 22.19 & 28.91 \\
\hline$P L V$ & 0.105 & 0.075 & 0.034 & 15.74 & 35.19 & 15.74 & 58.23 \\
\hline$V D$ & 0.000 & 0.000 & 0.000 & 12.69 & 65.29 & 22.02 & 16.27 \\
\hline$A A\left({ }^{\circ}\right)$ & 0.507 & 4.771 & 1.719 & 7.25 & 68.18 & 24.57 & 9.61 \\
\hline$B A\left({ }^{\circ}\right)$ & 22.105 & 13.537 & 9.101 & 54.49 & 27.21 & 18.30 & 66.69 \\
\hline 平均值 Mean & - & - & - & 22.55 & 51.55 & 24.62 & 30.78 \\
\hline
\end{tabular}

$A A$, 叶尖夹角; $B A$, 叶基夹角; $L A$, 叶面积; $L I$, 叶形指数; $L L$, 叶长; $L P$, 叶周长; $L W$, 叶宽; $L W R$, 叶长宽比; $P L$, 叶柄长; $P L V$, 一级侧脉数; $V D$, 叶脉 密度。

$A A$, apex angle; $B A$, base angle; $L A$, leaf area; $L I$, leaf index; $L L$, leaf length; $L P$, leaf perimeter; $L W$, leaf width; $L W R$, length to width ratio; $P L$, petiole length; $P L V$, primary lateral veins; $V D$, vein density.

表6 尾叶樱桃叶表型性状的主成分分析

Table 6 Principal component analysis of leaf phenotypic traits of Cerasus dielsiana

\begin{tabular}{|c|c|c|c|c|c|c|c|}
\hline \multirow{2}{*}{$\begin{array}{l}\text { 叶表型性状 } \\
\text { Leaf phenotypic trait }\end{array}$} & \multicolumn{3}{|c|}{ 主成分 Main components } & \multirow{2}{*}{$\begin{array}{l}\mid \begin{array}{l}\text { 叶表型性状 } \\
\text { Leaf phenotypic trait }\end{array}\end{array}$} & \multicolumn{3}{|c|}{ 主成分 Main components } \\
\hline & $\mathrm{PC} 1$ & $\mathrm{PC} 2$ & PC3 & & $\mathrm{PC} 1$ & $\mathrm{PC} 2$ & $\mathrm{PC} 3$ \\
\hline$L L(\mathrm{~mm})$ & 0.312 & -0.093 & -0.268 & $P L V$ & -0.020 & 0.071 & 0.021 \\
\hline$L W(\mathrm{~mm})$ & 0.367 & 0.149 & 0.163 & $V D$ & -0.332 & 0.182 & 0.302 \\
\hline$L W R$ & -0.055 & -0.242 & -0.431 & $A A\left(^{\circ}\right)$ & 0.109 & 0.604 & 0.244 \\
\hline$P L(\mathrm{~mm})$ & 0.186 & -0.667 & 0.650 & $B A\left(^{\circ}\right)$ & 0.022 & 0.102 & 0.242 \\
\hline$L A\left(\mathrm{~mm}^{2}\right)$ & 0.706 & 0.117 & -0.027 & 特征值 Eigenvalue & 0.092 & 0.015 & 0.011 \\
\hline$L P(\mathrm{~mm})$ & 0.327 & -0.039 & -0.139 & $\begin{array}{l}\text { 贡献率 } \\
\text { Variance contribution rate (\%) }\end{array}$ & 73.242 & 11.319 & 7.839 \\
\hline$L I$ & 0.051 & 0.195 & 0.251 & $\begin{array}{l}\text { 累计贡献率 } \\
\text { Cumulative Contribution rate (\%) }\end{array}$ & 73.242 & 84.561 & 92.400 \\
\hline
\end{tabular}

$A A$, 叶尖夹角; $B A$, 叶基夹角; $L A$, 叶面积; $L I$, 叶形指数; $L L$, 叶长; $L P$, 叶周长; $L W$, 叶宽; $L W R$, 叶长宽比; $P L$, 叶柄长; $P L V$, 一级侧脉数; $V D$, 叶脉 密度。

$A A$, apex angle; $B A$, base angle; $L A$, leaf area; $L I$, leaf index; $L L$, leaf length; $L P$, leaf perimeter; $L W$, leaf width; $L W R$, length to width ratio; $P L$, petiole length; $P L V$, primary lateral veins; $V D$, vein density.

www.plant-ecology.com 


\section{4 尾叶樱桃叶表型性状与地理、气候因子间的相} 关性

进一步对尾叶樱桃叶表型与采集地对应的地 理、气候因子做了相关性分析, 结果表明: 经度与 尾叶樱桃叶宽 $(r=-0.641)$ 、叶面积 $(r=-0.658)$ 呈显 著负相关关系, 而与一级侧脉数 $(r=0.659)$ 呈显著 正相关关系; 气温季节变化(bio4)分别与叶脉密度 $(r$ $=0.615)$ 和叶尖夹角 $(r=0.809)$ 呈显著或极显著正相 关关系, 与叶长 $(r=-0.631)$ 呈显著负相关关系; 最 湿季降水量(bio16)与叶长宽比 $(r=-0.600)$ 和叶形指 数 $(r=0.651)$ 呈显著负相关和正相关关系(表7)。

\section{5 基于尾叶樱桃叶表型性状和地理气候因子的} 主坐标分析与聚类分析

为了直观地显示尾叶樱桃种群间的亲缘关系, 选择尾叶樱桃 8 个种群代表前 3 个主成分的 10 个叶表 型性状联合对应的地理-气候因子, 进行主PCoA, 散点图分布结果与种群所在的实际地理位置有着良 好的对应关系(图1A)。进一步利用欧式平均距离,

表7 尾叶樱桃叶表型性状与地理、气候因子的Person相关性分析

Table 7 Person correlation analysis among leaf phenotypic traits and geographic-climatic factors of Cerasus dielsiana

\begin{tabular}{|c|c|c|c|c|c|c|c|}
\hline $\begin{array}{l}\text { 叶表型性状 } \\
\text { Leaf phenotypic trait }\end{array}$ & $\begin{array}{c}\text { 纬度 } \\
\text { Latitude (N) }\end{array}$ & $\begin{array}{c}\text { 经度 } \\
\text { Longitude (E) }\end{array}$ & $\begin{array}{c}\text { 海拔 } \\
\text { Altitude (m) }\end{array}$ & $\begin{array}{c}\text { 气温季节变化 } \\
\text { Temperature season- } \\
\text { ality bio4 }(S D \times 100)\end{array}$ & $\begin{array}{c}\text { 年降水量 } \\
\text { Annual precipita- } \\
\text { tion bio12 (mm) }\end{array}$ & $\begin{array}{c}\text { 最湿季降水量 } \\
\text { Precipitation of wettest } \\
\text { quarter bio16 (mm) }\end{array}$ & $\begin{array}{c}\text { 最暖季降水量 } \\
\text { Precipitation of warmest } \\
\text { quarter bio18 (mm) }\end{array}$ \\
\hline$L L(\mathrm{~mm})$ & 0.163 & -0.554 & 0.091 & $-0.613^{*}$ & -0.008 & 0.221 & -0.017 \\
\hline$L W(\mathrm{~mm})$ & 0.284 & $-0.641^{*}$ & 0.176 & 0.545 & 0.002 & 0.443 & 0.057 \\
\hline$L W R$ & -0.363 & 0.431 & -0.260 & -0.098 & -0.028 & $-0.600^{*}$ & -0.175 \\
\hline$P L(\mathrm{~mm})$ & -0.094 & -0.469 & 0.430 & 0.006 & -0.151 & 0.482 & 0.040 \\
\hline$L A\left(\mathrm{~mm}^{2}\right)$ & 0.227 & $-0.658^{*}$ & 0.162 & 0.551 & -0.016 & 0.459 & 0.037 \\
\hline$L P(\mathrm{~mm})$ & 0.184 & -0.593 & 0.141 & 0.585 & -0.009 & 0.315 & 0.008 \\
\hline$L I$ & 0.347 & -0.535 & 0.093 & 0.340 & 0.084 & $0.651^{*}$ & 0.206 \\
\hline$P L V$ & -0.248 & $0.659^{*}$ & 0.087 & -0.395 & 0.118 & -0.499 & 0.017 \\
\hline$V D$ & -0.220 & 0.590 & -0.035 & $0.651^{*}$ & 0.007 & -0.222 & 0.018 \\
\hline$A A\left({ }^{\circ}\right)$ & 0.324 & -0.249 & -0.429 & $0.809^{* *}$ & 0.303 & 0.127 & 0.181 \\
\hline$B A\left(^{\circ}\right)$ & -0.478 & 0.168 & 0.277 & -0.090 & 0.059 & -0.075 & 0.121 \\
\hline
\end{tabular}

$A A$, 叶尖夹角; $B A$, 叶基夹角; $L A$, 叶面积; $L I$, 叶形指数; $L L$, 叶长; $L P$, 叶周长; $L W$, 叶宽; $L W R$, 叶长宽比; $P L$, 叶柄长; $P L V$, 一级侧脉数; $V D$, 叶脉 密度。*表示在 $p<0.05$ 的显著水平, $* *$ 表示在 $p<0.01$ 的显著水平(经单尾检测)。

$A A$, apex angle; $B A$, base angle; $L A$, leaf area; $L I$, leaf index; $L L$, leaf length; $L P$, leaf perimeter; $L W$, leaf width; $L W R$, length to width ratio; $P L$, petiole length; $P L V$, primary lateral veins; $V D$, vein density. ${ }^{*}$ indicate significant correlation at the $p<0.05$ level, $* *$ indicate significant correlation at the $p<0.01$ level, assessed using one-tailed test.

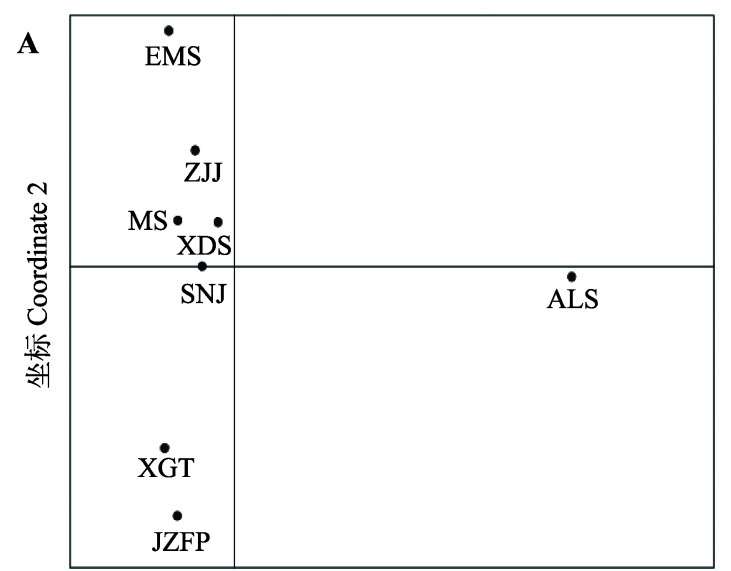

坐标 Coordinate 1

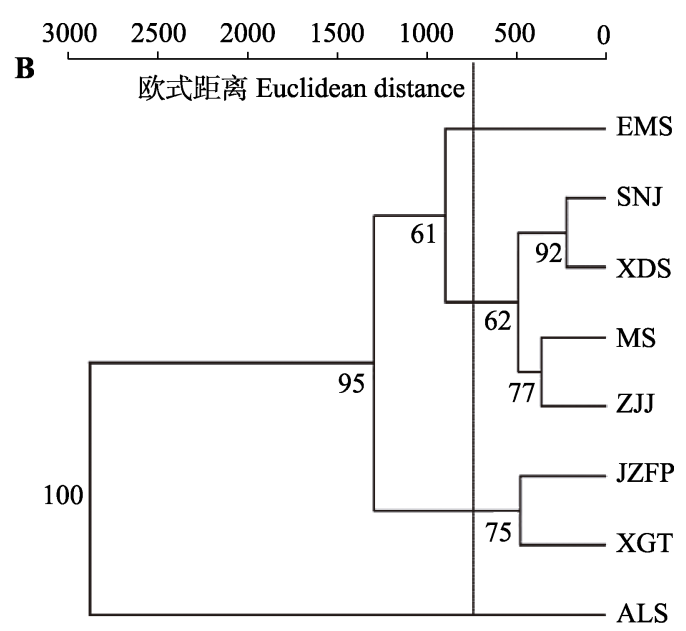

图1 基于叶表型性状和地理-气候因子的 8 个尾叶樱桃天然种群关系示意图。A, 主坐标分析输出结果。 $\mathbf{B}$, 非加权配对算术 平均法聚类分析输出结果。ALS, 台湾阿里山; EMS, 四川峨眉山; JZFP, 江西金竹飞瀑; MS, 湖南䒭山; SNJ, 湖北神农架; XDS, 湖北星斗山; XGT, 江西仙姑坛; ZJJ, 湖南张家界。

Fig. 1 Relationships between leaf phenotypic traits and geographic-climatic factors of the eight natural populations of Cerasus dielsiana. A, Output from principal coordinate analysis. B, Output from unweighted pair-group method with arithmetic mean cluster analysis. ALS, Mt. Ali, Taiwan. EMS, Mt. Emei, Sichuan; JZFP, Jinzhufeipu, Jiangxi; MS, Mt. Mangshan, Hunan; SNJ, Shennongjia, Hubei; XDS, Mt. Xingdou; Hubei; XGT, Xiangutan, Jiangxi; ZJJ, Zhangjiajie, Hunan. 
采用UPGMA法, 对尾叶樱桃 8 个种群进行聚类分析 (图1B)。在欧式距离500-1000阈值间，自上而下可 将 8 个种群分为 4 支: 四川峨眉山为第 1 分支; 湖北 神农架、湖北星斗山、湖南莽山和湖南张家界构成 第2分支; 江西金竹飞瀑和仙姑坛构成第3分支; 台 湾阿里山为第4分支。

\section{3 结论和讨论}

\section{1 尾叶樱桃叶表型变异的多样性}

植物表型多样性是遗传适应性和环境异质性长 期共同作用的结果, 植物表型变异程度越丰富, 越 能提高在不同环境下的适应程度(Verbeeck et al., 2014; Saenger \& West, 2018)。鉴于叶表型性状是最 直观的外在表现, 本研究基于尾叶樱桃天然分布区 采集的蜡叶标本进行统计分析, 大部分叶表型性状 在种群间和种群内的差异达到极显著 $(p<0.01)$ 水平, 体现了其叶片表型地理变异的丰富性; 变异系数最 大的为叶面积 $(C V=50.83 \%)$, 表明其性状可塑性最 大, 易受到个体发育和环境的双重影响; 变异系数 最小的为一级侧脉数 $(C V=7.96 \%)$, 方差分析在种 群间达到极显著差异而在种群内未达到显著性差异, 说明其在遗传上兼具种群间的变异性与物种间的稳 定性, 例如, 尾叶樱桃一级侧脉数平均在 12 对左右 (四川峨眉山最少有 11 对, 台湾阿里山和湖南䒭山 的侧脉最多均有 13 对), 在每个种群内部都是比较 稳定的, 与其他樱属植物——例如迎春樱桃 (C. discoidea) 有8-10对, 华中樱桃(C. conradinae) 有7-9对, 存在数量上的间断分布, 因此, 可以作为一个樱属 数量分类的重要参考指标。主成分分析结果(表5)进 一步说明, 在尾叶樱桃叶表型形态分化中, “大小性 状” 尤其是叶面积(特征值为0.706)起主要贡献作用, 也验证了变异系数的结果。从多重比较结果(表3)不 难看到, 不同种群的尾叶樱桃叶表型均有一定差异, 但这些差异在数量性状上均表现出一定的连续性。

\section{2 尾叶樱桃叶表型变异的来源与分化程度}

在巢氏方差中各层次上的方差分量百分比是说 明变异来源的一个重要指标, 也是计算表型分化系 数的主要依据(葛颂等, 1988)。11个尾叶樱桃种群平 均叶表型性状分化系数为 $30.78 \%$, 低于无患子 (Sapindus saponaria)( ᄀ松锋等, 2014, 62.21\%)、长柄 扁桃 (Amygdalus pedunculata)(柳江群等，2017, 45.90\%)、浙江柿(Diospyros glaucifolia)(井振华等,
2010, 40.15\%)、水青树(Tetracentron sinense)(李珊等, 2016, 46.69\%)、白檀(Symplocos paniculata)(杨艳, 2015, 59.16\%)、浙江楠(Phoebe chekiangensis)(陆云 峰等, 2018，41.43\%)等, 高于青钱柳(Cyclocarya paliurus)(余诚棋等, 2009, 20.54\%)、印楝(Azadirachta indica)(彭兴民等, 2012，11.89\%)等，与上述 物种相比, 由于分布碎片化和地理隔离导致尾叶樱 桃种群间的叶表型分化处于中等水平; 种群间的变 异能更加真实地反映地理与生殖隔离上的差异, 也 是种内多样性的重要组成部分(庞广昌和姜冬梅, 1995)。其中, 尾叶樱桃叶基夹角在各性状中的分化 系数 (66.69\%)最高, 种群间的方差分量百分比 (54.49\%) 是种群内 $(27.21 \%)$ 的 2 倍, 说明其变异在种 群间占优势; 而叶尖夹角的分化系数最低, 反映其 “尾尖”的特性最稳定; 叶表型种群内的平均方差分 量百分比(51.55\%)高于种群间的平均方差分量百分 比(22.55\%), 反映了种群内是变异的主要来源, 这 主要与其自身繁殖特性和基因流有关, 尾叶樱桃依 靠虫媒近距离传粉为主, 种群内杂交频繁, 加上自 花繁殖的能力强, 种子结实量大, 种群更新以实生 苗为主, 植株抗逆性强, 寿命长, 均有利于遗传变 异在种群中的保存; 而以果实为食的鸟兽与河流是 其种子扩散的长距离方式, 理论上有利于基因流的 扩散, 但通过实地观察, 尾叶樱桃种子通常在离开 母体后常温下 1 周左右就会逐渐丧生活能力, 其实 际的有效传播距离可能小于种群间的地理距离, 使 得种群间种子交流较少, 基于SSR 分子标记对近缘 樱属植物的研究结果(陈娇等, 2013)也得到相似的 结果。

\section{3 尾叶樱桃叶表型性状对地理、气候因子的响应}

植物生态学研究的一个重要内容就是确定和量 化各物种生态变量的主导维度, 并尝试阐释其机理 (Wright et al., 2007)。通过在较大尺度上针对特定植 物类群基于种群水平的表型变异的分析, 研究表型 性状与地理-气候间的关系, 往往可以更准确地揭 示植物变异格局及其生态响应策略(杨贺雨等, 2016; Meng et al., 2017)。相关性分析结果(表7)表明，尾叶 樱桃叶宽 $(r=-0.641)$ 、叶面积 $(r=-0.658)$ 和一级侧 脉数 $(r=0.659)$ 在地理空间上呈现以经度为主的单 一变异模式, 即自西向东随经度的增加, 8 个种群呈 现叶片变窄、叶面积变小和一级侧脉数增多的趋势。 这种在北半球随海洋向内陆的过渡中引起的地理经 
向变异规律也在麻栋(Quercus acutissima)(刘志龙等, 2011)、米老排(Mytilaria laosensis)(袁洁等, 2013)、 红椿(Toona ciliata)(汪洋等, 2018)等物种中有所报 道, 通常反映了水分和温度的梯度变化(Qian et al., 1998); 与此同时, 尾叶樱桃叶表型性状与气候因子 中的气温季节变化(bio4)和最湿季降水量(bio16)之 间存在统计上的相关性, 而与年降水量(bio12)和最 暖季降水量(bio18)无显著关系, 亦表明“气温变异 性”与 “展叶期降水量” 是驱动尾叶樱桃叶表型性状 变异的主要因子, 具体来说: 温差越大, 叶长越小、 叶尖夹角越大而叶脉密度越高; 展叶期降水量越大, 叶形越宽; 此外叶表型性状与纬度和海拔没有达到 显著相关性, 这可能受局部环境条件如土壤、坡度、 坡向等影响更大(杜会聪等, 2018), 有待日后从小尺 度上开展研究。

\section{4 尾叶樱桃叶表型性状变异的适应性机制}

植物形态特征变异往往具有适应性意义, 广阔 分布区、多样的生境与气候异质性, 为研究尾叶樱 桃叶表型地理变异模式提供了可能。从种群生态学 的角度看, 本研究选取的 8 个代表种群分布于我国 南方长江流域, 跨度范围为 $23.50^{\circ}-31.45^{\circ} \mathrm{N}$, $103.37^{\circ}-120.80^{\circ} \mathrm{E}$, 平均海拔范围839-1365 m, 其 中最西缘峨眉山种群属于横断山脉东部边缘的邛崃 山系, 平均海拔1 $365 \mathrm{~m}$, 在各种群中海拔分布最高; 而最东南缘的台湾种群, 与祖国大陆隔海相望, 所 在生境受亚热带季风气候影响(姜超等, 2017)程度 最大, 与高山、海峡等天然屏障共同加剧了局域生 境的隔离程度; 同时这也是尾叶樱桃叶各功能性状 相互协调的结果, 从全球尺度到区域尺度, 叶片大 小和结构与温度、水分、风速等气候条件之间存在 显著的相互关系, 譬如: 叶大小的形成机制与适应 价值普遍被认为是与叶的能量平衡变化有关 (Niinemets et al., 2010); 应用热成像技术和图像分 析技术对植物叶片样地数据定量研究显示, 在高 温、低风速环境下, 叶片宽度每减少 $1 \mathrm{~cm}$, 叶片表面 温度降低约 $2.1{ }^{\circ} \mathrm{C}$ (李永华等, 2018); 植物叶片变 小、变窄将有利于削弱自身的边界层阻力, 增强叶 边缘对水热与 $\mathrm{CO}_{2}$ 的传导能力, 进而降低呼吸和蒸 腾的成本, 使得相对小的叶片更利于生长在热、干、 高光和低养分的环境下(McDonald et al., 2003); 同 时, 作为叶片中的维管束组织和木质部成分, 叶脉 具有水分运输和支撑叶片的双重作用, 因此其密度
随温度的升高和光照强度的增加而增加, 以适应叶 片蒸腾作用的增强，随降水的增加而增加，以提高 叶片的抗打击能力(李东胜等, 2013)。综上所述, 我 们认为尾叶樱桃采取减小叶宽度, 缩小叶面积, 增 加叶脉密度的关联组合策略以适应亚热带东部地区 夏季强烈的高温高湿、强风降水与剧烈的干湿交替 的气候特征。

\section{5 尾叶樱桃天然种群保护与开发利用的建议与 展望}

尾叶樱桃作为优良的国产樱属代表性野生植物, 颇具观赏价值, 其市场潜在价值近年来已经逐渐被 人们所认识。然而当前尾叶樱桃天然种群正不断受 到人类经济活动的影响(如城市化、旅游开发), 面临 生境遭到破坏、资源减少的威胁; 就本研究而言, 8 个尾叶樱桃代表种群均位于人为干扰较少的自然保 护区或保护较好的景区，其他地区发现天然种群的 概率越来越低。由于种群内变异是尾叶樱桃种群变 异的主要来源，在开展种质资源收集、迁地或就地 保存与遗传改良时, 可适当减少抽样种群个体数, 增加核心种群内的取样密度，尤其是湖北、湖南、 江西三省的种群, 以增加遗传变异的丰富性; 同时, 种群间又存在中等程度的叶表型分化, 不同层次的 变异为优质种质资源和生物多样性保护提供了物质 基础(杨晓霞等, 2016; Humphrey et al., 2018)。结合 地理位置、主坐标与聚类分析结果看, 四川峨眉山 与台湾阿里山种群均远离尾叶樱桃多样性分布的中 心, 基因交流受限, 明显分化为两个独立分支, 值 得一提的是, 后者在中国植物志中英文版本中均未 记录, 为笔者近年来调查偶然发现, 属于尾叶樱桃 分布新记录。在种子资源就地保护或开发时，也应 该予以充分重视，通过采种播种、采穗扞插方式，加 强特征种群野生种质选择和收集。尾叶樱桃天然种 群表型地理变异格局上的差异是由其树种特性以及 适应性进化历史等因素所决定的, 从形态学水平上 对遗传变异进行探索, 但尚需整合生态地理学(生 态位模型)或分子亲缘地理学(谱系地理)等多方面的 研究, 以更深层次验证并阐明遗传变异格局的形成 机制，确定保护单元，有效的制订保护策略。

基金项目 江苏省林业三新工程项目 (LYSX[2015]17)、江苏省研究生科研创新计划项目 (KYCX17_0815)和2017年南京林业大学优秀博士 
学位论文创新基金(2169001)资助。

\section{参考文献}

Ali K (2018). Phenotypic characterization of Elaeagnus angustifolia using multivariate analysis. Industrial Crops and Products, 120, 155-161.

Chen J, Wang XR, Tang HR, Chen T, Huang XJ, Liang QB (2013). Assessment of genetic diversity and populations genetic structure in wild Chinese cherry from Sichuan Province using SSR markers. Acta Horticulturae Sinica, 40, 333-340. [陈娇, 王小蓉, 汤浩茹, 陈涛, 黄晓姣, 梁 勤彪 (2013). 基于SSR标记的四川野生中国樱桃遗传 多样性和居群遗传结构分析. 园艺学报, 40, 333-340.]

Dai ZC, Du DL, Si CC, Lin Y, Hao JL, Sun F (2009). A method to exactly measure the morphological quantity of leaf using scanner and Image $\mathrm{J}$ software. Guihaia, 29, 342-347. [戴志聪, 杜道林, 司春灿, 林英, 郝建良, 孙 凤 (2009). 用扫描仪及Image J软件精确测量叶片形态 数量特征的方法. 广西植物, 29, 342-347.]

Diao SF, Shao WH, Dong RX, Sun HG (2014). Phenotypic diversity in natural populations of Sapindus mukorossi based on fruit and seed traits. Acta Ecologica Sinica, 34, 1451-1460. [つ松锋, 邵文豪, 董汝湘, 孙洪刚 (2014). 基于种实性状的无患子天然群体表型多样性研究. 生 态学报, 34, 1451-1460.]

Du HC, Jiang YT, Zhang Y, Tian M, Duan GM (2018). Phenotypic trait variation of seed and fruit of wild populations of Chimonanthus praecox in Zhejiang Province and its relationship with soil related factors. Journal of Plant Resources and Environment, 27(2), 77-84. [杜会聪, 蒋雅 婷, 张莹, 田敏, 段国敏 (2018). 浙江省蜡梅野生种群 种实表型性状变异及其与土壤相关因子的关系. 植物 资源与环境学报, 27(2), 77-84.]

Fang YM, Ding YL (2016). Special Topic in Plant Biology. Chinese Forestry Publishing House, Beijing. [方炎明, 丁 雨龙. (2016). 植物生物学专题. 中国林业出版社, 北 京.]

Ge S, Wang MX, Chen YW (1988). An analysis of population genetic structure of masson pine by isozyme technique. Scientia Silvae Sinicae, 24, 399-409. [葛颂, 王明庥, 陈 岳武 (1988). 用同工酶研究马尾松群体的遗传结构. 林 业科学, 24, 399-409.]

Hijmans RJ, Guarino L, Cruz M, Rojas E (2001). Computer tools for spatial analysis of plant genetic resources data: 1 . DIVA-GIS. Plant Genetic Resources Newsletter, 127, $15-19$.

Humphrey PT, Gloss AG, Frazier J, Nelson-Dittrich AC, Faries S, Whiteman NK (2018). Heritable plant phenotypes track light and herbivory levels at fine spatial scales. Oecologia, 187, 427-445.

Jiang C, Tan K, Ren MX (2017). Effects of monsoon on distri- bution patterns of tropical plants in Asia patterns of tropical plants in Asia. Chinese Journal of Plant Ecology, 41, 1103-1112. [姜超，谭珂，任明迅（2017). 季风对亚洲 热带植物分布格局的影响. 植物生态学报, 41, 1103-1112.]

Jiang XB, Gong BC, Li DW, Wu KY, Zhao XM (2013). Variation analysis of phenotypic traits in natural population of Idesia polycarpa. Forest Research, 26, 113-117. [江锡兵, 龚榜初, 李大伟, 吴开云, 赵献民 (2013). 山桐子自然 群体表型性状变异分析. 林业科学研究, 26, 113-117.]

Jing ZH, Li H, Shao WH, Yue HF, Jiang JM (2010). Phenotypic diversity of natural populations in Diospyros glaucifolia. Bulletin of Botanical Research, 30, 325-331. [井振 华, 李皓, 邵文豪, 岳华峰, 姜景民 (2010). 浙江柿天 然群体表型多样性研究. 植物研究, 30, 325-331.]

Li B, Gu WC, Lu BM (2002). A study on phenotypic diversity of seeds and cones characteristics in Pinus bungeana. Biodiversity Science, 10，181-188. [李斌，顾万春，卢宝明 (2002). 白皮松天然群体种实性状表型多样性研究. 生 物多样性, 10, 181-188.]

Li DS, Shi ZM, Feng QH, Liu F (2013). Response of leaf morphometric traits of Quercus species to climate in the temperate zone of the North-South Transect of Eastern China. Chinese Journal of Plant Ecology, 37, 793-802. [李东胜, 史作民, 冯秋红, 刘峰 (2013). 中国东部南北样带暖温 带区栎属树种叶片形态性状对气候条件的响应. 植物 生态学报, 37, 793-802.]

Li M, Han HR, Kang FF, Ma QY (2005). Morphologic variation of leaves of Quercus liaotungensis Koidz. in Lingkong Mountain, Shanxi Province. Journal of Beijing Forestry University, 27(5), 10-16. [李梅, 韩海荣, 康峰 峰, 马钦彦 (2005). 山西灵空山辽东栋种群叶性状表型 变异研究. 北京林业大学学报, 27(5), 10-16.]

Li S, Gan XH, Han HY, Xu N, Hiu ZY, Chen Y, Deng XJ (2016). Leaf phenotypic traits of Tetracentron sinense, an endangered plant species. Forest Research, 29, 687-697. [李珊, 甘小洪, 憼宏艳, 许宁, 侯正扬, 陈杨, 邓晓娟 (2016). 濒危植物水青树叶的表型性状变异. 林业科学 研究, 29, 687-697.]

Li Y, Liu X, Ma J, Zhang X, Xu L (2018). Phenotypic variation in Phoebe bournei populations preserved in the primary distribution area. Journal of Forestry Research, 29(1), $35-44$.

Li YH, Li Z, Xin ZM, Liu MH, Li YL, Hao YG (2018). Effects of leaf shape plasticity on leaf surface temperature. Chinese Journal of Plant Ecology, 42, 202-208. [李永华, 李 臻, 辛智鸣, 刘明虎, 李艳丽, 郝玉光 (2018). 形态变 化对叶片表面温度的影响。植物生态学报, 42, 202-208.]

Liu JQ, Yin MY, Zuo SY, Yang SB (2017). Phenotypic variations in natural populations of Amygdalus pedunculata. Chinese Journal of Plant Ecology, 41, 1091-1102. [柳江

www.plant-ecology.com 
群, 尹明宇, 左丝雨, 杨绍斌 (2017). 长柄扁桃天然种 群表型变异. 植物生态学报, 41, 1091-1102.]

Liu ZL, Yu MK, Ma Y, Tang LZ, Fang SZ (2011). A trend surface analysis of geographic variation in the triats of seeds and seedlings from different Quercus acutissima provenances. Acta Ecologica Sinica, 31, 6796-6804. [刘 志龙, 虞木奎, 马跃, 唐罗忠, 方升佐 (2011). 不同种 源麻栎种子和苗木性状地理变异趋势面分析. 生态学 报, 31, 6796-6804.]

Lu YF, Pei NC, Zhu YJ, Bai ZJ, Yang AN, Zhang JH, Lou LH, Tong ZK (2018). Community structure and leaf trait diversity in a vulnerable species, Phoebe chekiangensis. Chinese Journal of Applied Ecology, 29, 2101-2110. [陆 云峰, 裴男才, 朱亚军, 柏志埥, 杨安娜, 张俊红, 楼炉 焕, 童再康 (2018). 渐危植物浙江楠群落结构及叶片性 状多样性. 应用生态学报, 29, 2101-2110.]

McDonald PG, Fonseca CR, Overton JM, Westoby M (2003). Leaf-size divergence along rainfall and soil-nutrient gradients: Is the method of size reduction common among clades? Functional Ecology, 17, 50-57.

Meng H, Wei X, Franklin SB, Wu H, Jiang M (2017). Geographical variation and the role of climate in leaf traits of a relict tree species across its distribution in China. Plant Biology, 19, 522-561.

Niinemets Ü, Portsmuth A, Tobias M (2010). Leaf size modifies support biomass distribution among stems, petioles and mid-ribs in temperate plants. New Phytologist, 171, 91-104.

Pang GC, Jiang DM (1995). Population genetic diversity and data analysis. Scientia Silvae Sinicae, 31, 543-550. [庞广 昌, 姜冬梅 (1995). 群体遗传多样性和数据分析. 林业 科学, 31, 543-550.]

Peng XM, Wu JC, Zheng YX, Zhang YP, Li GQ (2012). Phenotypic variation in cultivated populations of Azadirachta indica in Yunnan, China. Chinese Journal of Plant Ecology, 36, 560-571. [彭兴民, 吴疆羽, 郑益兴, 张燕平, 李根前 (2012). 云南引种印楝实生种群的表型变异. 植 物生态学报, 36, 560-571.]

Qian H, Klinka K, Kayahara G (1998). Longitudinal patterns of plant diversity in the North American boreal forest. Plant Ecology, 138, 161-178.

Saenger P, West PW (2018) Phenotypic variation of the mangrove species Avicennia marina (Forssk.) Vierh. from seven provenances around Australia. Aquatic Botany, 149, 28-32.

She CQ, Yang WX, Fang SZ, Shang XL (2009). Phenotypic diversity of natural Cyclocarya paliurus populations seed traits. Chinese Journal of Applied Ecology, 20, 2351-2356. [余诚棋, 杨万霞, 方升佐, 尚旭岗 (2009). 青钱柳天然群体种子性状表型多样性. 应用生态学报, 20, 2351-2356.]

Verbeeck H, Betehndoh E, Maes WH, Hubau W, Kearsley E,
Buggenhout L, Hufkens K, Huygens D, van Acker J, Beeckman H, Mweru JPM, Boeckx P, Steppe K (2014). Functional leaf trait diversity of 10 tree species in Congolese secondary tropical forest. Journal of Tropical Forest Science, 26, 409-419.

Wang MZ, Bu XQ, Li L, Dong BC, Li HL, Yu FH (2018). Constraints on the evolution of phenotypic plasticity in the clonal plant Hydrocotyle vulgaris. Journal of Evolutionary Biology, 31, 1006-1017.

Wang XR (2014). An Illustrated Monograph of Cherry Cultivars in China. Science Press, Beijing. [王贤荣 (2014). 中 国樱花品种图志. 科学出版社, 北京.]

Wang XR, Xie CP, Yi XG, Xiang QB (2007). Study on the morphological variations of Cerasus subhirtella var. ascendens in different populations. Bulletin of Botanical Research，27，746-752. [王贤荣，谢春平，伊贤贵，向其柏 (2007). 不同居群野生早樱形态变异研究. 植物研究, 27, 746-752.]

Wang Y, Tian YE, Gan XY, Song F (2018). Geographic trend surface analysis of phenotypic variance of Toona ciliata in natural populations of Hubei. Journal of Forest and Environment, 28, 309-317. [汪洋, 田玉娥, 甘小燕, 宋菲 (2018). 湖北红椿天然居群表型变异地理趋势面研究. 森林与环境学报, 28, 309-317.]

Wright IJ, Ackerly DD, Bongers F, Harms KE, IbarraManriquez G, Martinez-Ramos M, Mazer SJ, MullerLandau, HC, Paz H, Pitman NCA, Poorter L, Silman MR, Vriesendorp CF, Webb CO, Westoby M, Wright SJ (2007). Relationships among ecologically important dimensions of plant trait variation in seven neotropical forests. Annals of Botany, 99, 1003-1015.

Yang HY, Wei HY, Sang MJ, Shang ZH, Mao YJ, Wang XR, Liu F, Gu W (2016). Phenotypic plasticity of Schisandra sphenanthera leaf and the effect of environmental factors on leaf phenotype. Chinese Bulletin of Botany, 51, 322-334. [杨贺雨, 卫海燕, 桑满杰, 尚忠慧, 毛亚娟, 王小荵，刘芳，顾蔚 (2016). 华中五味子叶表型可塑性 及环境因子对叶表型的影响. 植物学报, 51, 322-334.]

Yang XX, Leng PS, Zheng J, Hu ZH, Liu XY, Yang XH, Dou DQ (2016). Variation of phenotypic traits of seed and seedling of different provenances and their correlations with geographic-climatic factors Syringa reticulata subsp amurensis from different provenances and their correlations with geographic-climatic factors. Journal of Plant Resources and Environment, 25(3), 80-89. [杨晓霞, 冷平 生, 郑健, 胡增辉, 刘学娅, 杨晓红, 窦德泉 (2016). 暴 马丁香不同种源种子和幼苗的表型性状变异及其与地 理-气候因子的相关性. 植物资源与环境学报, 25(3), 80-89.]

Yang Y, Liu J, Yin X, Chen JZ, Jiang LJ (2015). Phenotypic diversity and environment relations in Symplocos paniculata of Hunan. Journal of Plant Genetic Resources, 16, 
80-86. [杨艳, 刘强, 尹翔, 陈景震, 蒋丽娟 (2015). 湖 南白檀居群形态多样性及与环境的相关性. 植物遗传 资源学报, 16, 80-86.]

Yuan J, Yin GT, Yang JC, Li RS, Zou WT (2013). Phenotypic diversity of seed and fruits in natural populations of Mytilaria laosensis. Chinese Journal of Tropical Crops, 34, 2057-2062. [袁洁, 尹光天, 杨锦昌, 李荣生, 邹文涛 (2013). 米老排天然群体的种实表型变异研究初报. 热 带作物学报, 34, 2057-2062.]

Zhang WX, Fan JJ, Yang P, Zhou T, Pu J, Cao FL (2017). Analysis of phenotypic characteristics of the Malus 'Purple Prince' half-sib progenies at the seedling stage. Journal of Nanjing Forestry University (Natural Science Edi- tion), 41(6), 33-40. [张往祥, 范俊俊, 杨萍, 周婷, 浦静, 曹福亮 (2017). “紫王子”海棠半同胞家系苗期表型特征 分析. 南京林业大学学报(自然科学版), 41(6), 33-40.]

Zhu H, You LX, Li YF, Wang HC, Wang XR (2017). Modeling the geographical distribution pattern and climatic limited factors of Cerasus schneideriana. Journal of Tropical and Subtropical Botany, 25, 315-322. [朱弘, 尤禄祥, 李涌 福, 王华辰, 王贤荣 (2017). 浙闽樱桃地理分布模拟及 气候限制因子分析. 热带亚热带植物学报, 25 , 315-322.]

责任编委: 王国宏 责任编辑: 李 敏 